\title{
Are Nurse Injectors the New Norm?
}

\author{
Neil S. Sadick
}

Received: 24 July 2014/ Accepted: 1 August 2014/Published online: 5 September 2014

(C) Springer Science+Business Media New York and International Society of Aesthetic Plastic Surgery 2014

Level of Evidence $V$ This journal requires that authors assign a level of evidence to each article. For a full description of these Evidence-Based Medicine ratings, please refer to the Table of Contents or the online Instructions to Authors at www.springer.com/00266.

The article entitled "Are Nurse Injectors the New Norm?" asks a somewhat controversial but realistic question: Who is delivering filler treatments in 2014 in the US and perhaps on a global basis as well? It also brings up several other questions as well: What is the watershed level of training and experience that would qualify an individual to be considered an expert injector? What is in the best interest of the patient, i.e., safety and reliable outcomes? What role does economics play? What role does potential professional bias play in the overall equation? Who is truly the most qualified to perform filler injection procedures is the subject of the present article.

It is generally believed that education, training, and experience are the major factors associated with becoming a competent filler injector. Certainly dermatologists and plastic and cosmetic surgeons have the greatest exposure to the procedure during their training and educational programs in their core specialties. They also have more training in the anatomical aspects of the aging face which translates into a potentially better aesthetic skill set in this regard. That is not to say that other individuals can not obtain this expertise via the experience route. The reality is that in many plastic surgery practices and select dermatology settings, physician assistants and nurse practitioners perform filler injections with expertise. However, the general consensus is that dermatologists and plastic surgeons have the most extensive training in this area, with the most reliable outcomes and best safety profile. This is a general rule and should be understood as such. Most other individuals e.g., physician extenders and noncore physicians, get their training through experience or with handson courses and then build up their experience with time. Experience plays a major role in all procedures and that is why there is a general consensus that registered nurses are better at administering vaccinations, which they perform on a more regular basis.

Finally, a major factor in why dermatologists and plastic surgeons may be the best providers in this setting is that they are most capable to recognize and manage any complications. Core specialists are best trained in this important aspect of filler care as significant and more frequent complications are recognized. In terms of patient safety and severe outcomes, this is perhaps the most important key factor.

In conclusion, many providers will continue to offer filler procedures. It is up to the consumer to decide where they choose to have these services performed. The majority of the physicians who provide these services have the best outcomes and the highest safety levels regardless of the specialty.

Conflict of interest The author has no conflicts of interest to disclose.
N. S. Sadick $(\bowtie)$

Department of Dermatology, Weill Cornell Medical College,

New York, NY, USA

e-mail: nssderm@sadickdermatology.com 